\title{
El programa para dejar de fumar "on line" del Ayuntamiento de Madrid. Un estudio exploratorio
}

\author{
José Javier Moreno Arnedillo \\ Psicólogo. Asesor técnico. Servicio de Prevención y Promoción de la Salud. Madrid Salud. Ayuntamiento de Madrid \\ Enviar correspondencia a: \\ José Javier Moreno Arnedillo. Centro de Promoción de Hábitos Saludables. C/ Navas de Tolosa 10. 28013-Madrid. E-mail: morenoajj@munimadrid.es
}

Recibido: 27 de abril de 2006 Aceptado: 7 de agosto de 2006.

\section{RESUMEN}

Una parte sustancial de los fumadores son capaces de abandonar el uso del tabaco simplemente con una guía u orientación mínima y sin necesidad de programas costosos de tipo presencial. De entre las estrategias utilizables para proveer guía y orientación para dejar de fumar, el uso de internet combina ventajas propias de los medios de comunicación de masas (algunas de ellas, además, optimizadas) junto con ventajas propias de la interacción cara a cara. En este artículo se presentan los objetivos, características, funcionamiento y resultados del Programa para Dejar de Fumar "on line" del Ayuntamiento de Madrid, un sistema que combina la interacción automatizada con el usuario y la consulta personal vía correo electrónico. Después de 9 meses en la red, y sobre 4865 sujetos, 868 (17,8 \%) declararon permanecer sin fumar tras el período de tratamiento. En un análisis de regresión logística posterior se identificaron cuatro variables como predictoras de los resultados: tipo de procedimiento utilizado, género, expectativas de autoeficacia y haber permanecido anteriormente más de 3 meses sin fumar. Se comprueba el coste-efectividad de este tipo de procedimientos, se plantea alguna hipótesis sobre aspectos clave que condicionan su efectividad y se sugieren algunas posibles mejoras.

Palabras clave: tabaco, fumar, internet, tabaquismo, tratamiento.

\begin{abstract}
A substantial proportion of smokers is able to abandon the use of tobacco simply with minimal guidance or direction without the need for expensive programmes involving physical participation. Among the strategies utilizable in providing guidance and direction in giving up smoking, the use of the Internet combines all the specific advantages of the mass media (some, also optimised) together with the particular advantages of face-to-face interaction. This paper presents the objectives, characteristics, performance and results of the Madrid City Council on-line programme, Giving up Smoking, a system which combines both automated interaction with the user and personal consultations via e-mail. After 9 months on the net, 868 (17.8\%) of 4865 subjects declared they were not smoking after the treatment period. In a posterior logistic regression analysis, four variables were identified as predicting the results: type of procedure employed, gender, self-efficacy expectative, and having previously been more than 3 months without smoking. The cost-effectiveness of procedures of this kind is an important issue, and some hypothesis on key issues determining its effectiveness are presented, and some possible improvements suggested.
\end{abstract}

Key words: tobacco, smoking, Internet, on line, treatment.

\section{INTRODUCCIÓN}

$\mathbf{S}$ egún la Encuesta de Salud de la Ciudad de Madrid (en prensa), un 27,3\% de los habitantes de esta ciudad mayores de 16 años son fumadores; en el conjunto del Estado, los datos de la Encuesta Nacional de Salud referidos al año 2003 situaban este porcentaje en el $32 \%$; el contraste entre ambos datos puede ser reflejo de diferencias geográficas o, quizá, de una tendencia temporal hacia la disminución de la cantidad de fumadores asociada, tal vez, a los cambios legislativos recientes. En cualquier caso, entre 10 y 11 millones de españoles son fumadores. De ellos, una proporción muy elevada desea dejar de fumar y estaría dispuesta a realizar un serio intento para conseguirlo.

Resulta muy difícil, por no decir imposible y probablemente innecesario que estos varios millones de personas reciban tratamientos costosos de tipo multicomponente en formato clínico tradicional. Con mucha probabilidad, buena parte de estas personas serían capaces de abandonar el consumo de tabaco simplemente con una guía o una orientación mínima. 
No hay que olvidar que una proporción de exfumadores que Fiore, Novotny, Pierce, Giovino, Hatziandreu et al. (1990) situaban en el 90 \% abandonaron el tabaco sin necesidad de acudir a ningún profesional. En otros casos, los fumadores no desean o no disponen de tiempo para acudir a unidades de tratamiento, o simplemente estas no existen en su entorno.

Por estos motivos, una de las estrategias más elementales y consolidadas en la lucha contra la dependencia tabáquica es la elaboración y difusión de guías de autoayuda. Estas guías existen desde hace décadas en soporte de papel, y contienen básicamente la serie de pautas que el fumador debe seguir para abandonar el consumo y no recaer. Un ejemplo es la guía editada recientemente en nuestro país por el Ministerio de Sanidad y Consumo (2005). Una revisión de la historia, las características y la eficacia de estos procedimientos puede encontrarse en Míguez (2004).

Sin embargo, los procedimientos de autoayuda tradicionales basados en material escrito presentan serias limitaciones:

- Es difícil evaluar su efectividad real, por cuanto por lo general no implican registro de datos ni control alguno sobre los usuarios que las utilizan.

- Abordan aspectos generales, orientados hacia un fumador-tipo único; no permiten individualizar las pautas que el fumador recibe.

- Son costosas de elaborar, distribuir y destruir, por estar realizadas en papel.

- Están donde están; el acceso a ellas depende de que se distribuyan de modo eficiente, y en ningún caso son accesibles al mismo tiempo desde el domicilio, el trabajo, el lugar de vacaciones...

- No permiten incluir nuevos contenidos o modificar los existentes una vez que han sido elaboradas, salvo que se realice una nueva edición que en todo caso implica un proceso lento y costoso.

- No permiten la bidireccionalidad, es decir, el ajuste de las pautas en función del feedback proporcionado por el usuario, lo cual redunda en su efectividad; por lo mismo, tampoco permite el refuerzo de los pasos intermedios a medida que el usuario avanza.

- Plantean toda la información "de golpe", y no dividida en unidades pequeñas, lo cual constituye una estrategia básica de mejora del aprendizaje.

- No permiten el uso de medios sonoros o de animaciones.

Otros procedimientos que salvan algunas de estas limitaciones, y que se ha utilizado frecuentemente en los últimos años con el objetivo de combinar el acceso a un número elevado de usuarios al mismo tiempo que un "feedback" personalizado, son el correo y la combinación de correo y teléfono. Un ejemplo es el programa de tratamiento por correo de la Unidad de Tabaquismo de la Universidad de Santiago de Compostela (www.usc.es/pdf2001). Estos medios permiten personalizar los contenidos de la intervención en mayor medida que los manuales de autoayuda, y sobre todo permiten que exista un "feedback" individual entre terapeuta y usuario. Existen trabajos en nuestro país cuyos datos apuntan precisamente a una mayor efectividad cuando junto con el material impreso existe "feedback", ya sea escrito (Becoña y Vázquez, 2001) o telefónico en su modalidad proactiva, es decir, cuando la llamada parte del propio equipo terapéutico a intervalos programados en lugar de esperar a que sea el usuario quien llame (Miguez et al., 2002).

No obstante, parece obvio que, si se trata de combinar las ventajas de los medios de comunicación de masas junto con las propias de las interacciones cara a cara entre profesional y usuario, Internet ofrece posibilidades incomparables:

- La información es accesible a un gran número de usuarios.

- La información es accesible en cualquier lugar y a cualquier hora del día o de la noche. Al mismo tiempo, no requiere canales o sistemas de distribución costosos, lentos o demorados.

- La respuesta que el usuario obtiene del programa es siempre inmediata; sabemos que la inmediatez del feedback o del refuerzo optimiza el aprendizaje.

- La eficacia de las intervenciones es evaluable porque es fácil establecer sistemas automatizados de registro y seguimiento de los usuarios.

- Internet permite la interacción entre sistema y usuario, que ya no es un mero receptor pasivo de información. Sabemos que los aprendizajes que implican actividad por parte de los usuarios son más eficaces. Además, este feedback del usuario permite modular la intervención, que de este modo puede adaptarse a determinados parámetros, incluso de modo automatizado a través de algoritmos de decisión, y por lo tanto ser más eficaz.

- No se requiere papel ni ningún otro soporte costoso.

- Los contenidos pueden ser permanentemente actualizados de modo muy sencillo.

- Los procedimientos automatizados pueden ser complementados con intervenciones más personalizadas a través del correo electrónico o incluso a través de sistemas de contacto en tiempo real que se aproximan notablemente a la situación de la terapia cara a cara. Igualmente pueden combinarse con procedimientos menos estructurados de apoyo mutuo como chats o foros.

- El sistema mantiene un control no sólo de los contenidos, sino también de su organización, de modo que pueden ser estructurados en unidades pequeñas de aprendizaje. 
- El material escrito o gráfico no es el único formato posible; cabe también el uso de sonidos, videos o animaciones.

A la luz de todas esta ventajas, es fácil comprender por qué han aparecido en los últimos tiempos distintos tipos de programas orientados a la promoción de conductas saludables o al tratamiento de problemas de conducta que utilizan Internet, y que han dado lugar a una verdadera subespecialización de la psicología clínica en lo que se ha llamado "ciberterapia", "terapia on line", "e-therapy", "telepsicología" o "telepsiquiatría"; esta subespecialización se fundamenta además en determinados principios teóricos y destrezas específicas (Fenichel, M., Suler, J., Barak, A., Zelvin, E., Jones, G., Munro, K., et al. , 2002).

Estas intervenciones varían en su grado de automatización y de consumo de recursos, oscilando desde el uso de programas que interactúan por sí solos con el usuario a través de algoritmos de respuestas hasta la interacción entre individuos en tiempo real en la que Internet es sólo un medio de contacto. Asimismo, las intervenciones varían en cuanto al alcance de las mismas desde contactos aislados que incluyen información o evaluación y feedback mínimos hasta intervenciones estructuradas con contactos a largo plazo. En todo caso, existe evidencia de la efectividad de estos programas en áreas como el tabaquismo, el abuso de alcohol, el trastorno de pánico, la obesidad o los problemas de ansiedad o depresión. (Copeland y Martin, 2004).

En este artículo se presenta una descripción de los objetivos, los procedimientos y los resultados de un programa para dejar de fumar a través de Internet implementado en Abril de 2005 por el organismo autónomo Madrid Salud, dependiente del Ayuntamiento de Madrid, y accesible en la dirección www. munimadrid.es/dejardefumar.

\section{MATERIALY MÉTODOS}

Se presenta un estudio descriptivo de las características y de los resultados del programa para dejar de fumar "on line" de Madrid Salud (Ayuntamiento de Madrid) y un estudio exploratorio de las variables asociadas a los resultados mediante un análisis de regresión logística.

\section{Objetivos del programa}

El programa pretende facilitar el acceso a un recurso que ayude a dejar de fumar al mayor número posible de usuarios. Por lo tanto, y en la línea de los procedimientos de autoayuda y en contra de los procedimientos de formato clínico tradicional, se prefiere un porcentaje menor de éxitos pero sobre un número mayor de destinatarios y a un bajo coste. Obviamente, este matiz es de suma importancia a la hora de valorar los resultados.

\section{Sujetos participantes}

En el estudio participan 4865 sujetos. Este número constituye el total de los sujetos inscritos que no abandonaron ni fueron dados de baja por el sistema durante las dos primeras semanas, existentes en la base de datos 9 meses después del inicio. No se establece por lo tanto ningún procedimiento de selección posterior. Sus principales características aparecen en la tabla 1.

Tabla 1. Principales datos descriptivos de los sujetos participantes.

\begin{tabular}{|c|c|}
\hline Número de sujetos & 4865 \\
\hline Distribución por sexo & $\begin{array}{ll}43,1 \% & \text { varones } \\
50,3 \% & \text { mujeres } \\
6,6 \% & \text { no declarado }\end{array}$ \\
\hline Distribución por nivel educativo & $\begin{array}{ll}3,4 \% & \text { sin estudios } \\
5,5 \% & \text { primarios } \\
14,7 \% & \text { secundarios } \\
30,8 \% & \text { medios } \\
45,5 \% & \text { superiores } \\
\end{array}$ \\
\hline Distribución por edad & $\begin{array}{ll}1,8 \% & \text { Menos de } 21 \text { años } \\
29,0 \% & \text { Entre } 21 \text { y } 30 \text { años } \\
35,1 \% & \text { Entre } 31 \text { y } 40 \text { años } \\
25,3 \% & \text { Entre } 41 \text { y } 50 \text { años } \\
7,4 \% & \text { Entre } 51 \text { y } 60 \text { años } \\
1,3 \% & \text { Más de } 60 \text { años } \\
\end{array}$ \\
\hline Puntuación Test de Fagerstrom & $\begin{array}{l}\text { Media }=5,06 \\
\text { Desv. Típ. }=2.63\end{array}$ \\
\hline Puntuación expectativas de autoeficacia (rango 0 - 100) & $\begin{array}{l}\text { Media }=60.6 \\
\text { Desv. Típ. }=26.17\end{array}$ \\
\hline Distribución por fase del proceso de cambio & $\begin{array}{l}13,9 \% \text { Precontemplación } \\
12,3 \% \text { Contemplación } \\
14,7 \% \text { Preparación } \\
59,1 \% \text { Actuación }\end{array}$ \\
\hline Distribución por pauta de tratamiento asignada & $\begin{array}{ll}10,3 \% & \text { Tratamiento conductual + TSN a dosis altas } \\
10,7 \% & \text { Tratamiento conductual + TSN a dosis estándar } \\
10,0 \% & \text { Tratamiento conductual + TSN a dosis bajas } \\
69,0 \% & \text { Tratamiento conductual + Reducción Gradual }\end{array}$ \\
\hline
\end{tabular}




\section{Contenidos básicos del programa}

Se trata de un programa multicomponente y estructurado, basado en el programa presencial existente desde 1998 en los centros Madrid Salud (Ayuntamiento de Madrid), así como en distintas guías impresas de autoaplicación (como la de Becoña (1997)). Este programa sigue las recomendaciones contenidas en las principales guías, revisiones y metaanálisis. Entre ellas cabe citar las revisiones de la Cochrane Collaboration (Lancaster y Stead, 2003a; Stead y Lancaster, 2005; Silagy, Lancaster, Stead, Mant y Fowler, 2005; Hajek y Stead, 2003; Lancaster y Stead, 2003b; Lancaster y Stead, 2005), la Guía para la práctica clínica de la Agency for Healthcare Research and Quality (AHRQ) (Fiore, M. C., Bailey, W. C., Cohen, S. J., Dorfman, S. F., Goldstein, M. G., Gritz, E. R. et al., 2000; Tsoh, J. Y. y McClure, J. B., 1997), la Guía para tratamiento de la dependencia de la nicotina de la Health Education Authority (Reino Unido) (Raw, M., McNeill, A. y West, R., 1998), y la Guía del Comité Nacional para la Prevención del Tabaquismo (España) (Comité Nacional para la Prevención del Tabaquismo, 2001), entre otras.

Consecuentemente, el programa incluye distintos componentes que se orientan a distintos objetivos. Estos componentes son planteados al usuario en dos fases: la primera, durante las dos semanas posteriores al día elegido para empezar (con parches de nicotina o con reducción de ingesta de nicotina); la segunda, a partir de la tercera semana.

Los objetivos y componentes que se abordan en la primera fase (primeras dos semanas) son:

- Optimizar y mantener la motivación. Incluye la elaboración por parte del usuario de listas de beneficios que se esperan obtener por dejar de fumar, junto con sugerencias para ayudar a realizar esta tarea ("listas de otros") e instrucciones para revisar y ampliar la lista una vez elaborada. Incluye también información sobre la nocividad del tabaco. Además, se presta especial atención al refuerzo verbal por los avances, tanto programado en la web como en todos los contactos por correo electrónico que se mantienen con el usuario.

- Establecer expectativas realistas sobre el proceso. Las expectativas inadecuadas o poco realistas condicionan poderosamente la implicación del usuario en el programa en la medida en que su no cumplimiento puede generar frustración y abandono. Por este motivo el programa incluye instrucciones orientadas a establecer que:

El objetivo del programa es dejar de fumar del todo y para siempre. No consiste en una reducción del consumo ni en un abandono temporal.
El objetivo del programa es aprender a controlar el deseo de fumar, no eliminarlo.

Por lo tanto, durante bastante tiempo es normal tener ganas de fumar, si bien en conjunto son progresivamente menos intensas y frecuentes, y siempre son controlables.

Dejar de fumar requiere un esfuerzo activo por parte del usuario (no existen "varitas mágicas") que además debe ejecutarse de modo metódico y planificado. No sirve la mera apelación a la fuerza de voluntad. Realizar las tareas que se piden a lo largo del programa es, por lo tanto, una variable que predice buenos resultados.

- Reducir la intensidad del síndrome de abstinencia nicotínico. Este objetivo se consigue mediante apoyo farmacológico (parches de nicotina) o procedimientos de reducción gradual de la ingesta de nicotina y alquitrán. Ambos procedimientos son ofertados al usuario y su viabilidad es valorada por el programa. En el caso del uso de nicotina transdérmica se usan las pautas descritas en la tabla 2; en el caso de la reducción gradual de la ingesta de nicotina y alquitrán, se usa el procedimiento descrito por Becoña (1994), y que básicamente consiste en la indicación de fumar durante una semana una nueva marca de cigarrillos que contenga aproximadamente un $65 \%$ de la concentración de nicotina de la marca original, seguida de otra semana durante la cual se debe fumar otra marca que contenga en torno a un $35 \%$ de la concentración de nicotina original, al término de la cual se debe dejar de fumar completamente. La efectividad de ambos métodos cuenta con sólido apoyo empírico (Gil Roales-Nieto, 2003).

- Crear una red social de compromiso y apoyo en el entorno del fumador. El fumador es instado a que comunique a su familia, amistades o compañeros de trabajo su participación en el programa. De este modo, se pretende conseguir su apoyo y, también, aumentar el compromiso del usuario con su decisión en la medida en que este ya no queda reducido a la interacción con el sistema sino que incluye igualmente a su entorno social.

- Organizar el entorno y los hábitos de modo que favorezcan el mantenimiento de la abstinencia, mediante pautas de control estimular.

- Aprender a afrontar el deseo cuando aparece. El fumador es instruido para focalizar su atención en estímulos distractores y utilizar el lenguaje de modo que favorezca el control del deseo.

- Controlar la ansiedad, mediante instrucciones de respiración profunda y relajante.

- Autorreforzarse por el mantenimiento de la abstinencia. El usuario es instruido para que guarde dia- 
riamente en una hucha el dinero que ahorra por no fumar, y se premie con un pequeño regalo semanal.

Los objetivos y componentes que se abordan durante la segunda fase (a partir de la tercera semana) consisten en:

- Controlar la ansiedad asociada al abandono del tabaco, continuando con las instrucciones de respiración profunda.

- Aprender a identificar y cuestionar ideas irracionales y excusas que puedan favorecer la recaída. El usuario recibe información sobre los principales pensamientos que actúan como disparadores de la recaída, y debe ejercitarse en el cuestionamiento de los mismos y su sustitución por pensamientos alternativos.

- Promover la habituación y la autoeficacia del exfumador al afrontamiento de situaciones condicionadas al deseo de fumar mediante técnicas de exposición. Se explica al exfumador que la habituación y las expectativas de autoeficacia implican la exposición progresiva a situaciones previamente asociadas al consumo de tabaco, y se le anima a que de modo gradual vaya afrontando estas situaciones.

- Implementar nuevos hábitos que favorezcan la abstinencia (alimentación, ejercicio físico, aficiones e intereses). Se subraya que la abstinencia a largo plazo se ve favorecida por la puesta en marcha de nuevos hábitos y estilos de vida más saludables y gratificantes, y se instruye para que se introduzcan cambios en esta línea.

\section{Procedimiento}

Se diseñó un sistema informático que incluye una página web (www.munimadrid.es/dejardefumar) y un buzón de correo electrónico para consultas. Todas las consultas son respondidas por un psicólogo experto en tabaquismo. Salvo la participación de este profesional y las actividades propias de mantenimiento del sistema informático, todas la interacción del programa con el usuario es automática y no implica coste alguno adicional de recursos humanos.

Cuando el usuario accede a la web encuentra un menú con las siguientes opciones:

- ¿Qué somos/qué hacemos?

- Inscripción al Programa

- Acceso Usuarios Registrados

- Preguntas Frecuentes. (FAQ)

- Contacta con nosotros
- Agregar a favoritos

- Página de Inicio

- Más información sobre la dependencia del tabaco

\section{Bloque 1: información y motivación}

Las tres primeras opciones constituyen la vía de entrada al protocolo de tratamiento. Normalmente, quien accede por primera vez elige la primera opción ("¿Qué somos / qué hacemos?") y llega así al material de información sobre el programa y de motivación para participar, del que luego es remitido a la ficha de inscripción, en caso de que decida inscribirse. También es posible ampliar la información desde los enlaces "Preguntas frecuentes (FAQ)" y "Más información sobre la dependencia del tabaco".

En este primer bloque, que es de acceso libre, se proporciona información acerca de las siguientes cuestiones:

- La existencia del programa y sus características esenciales (destinatarios, gratuidad, interactividad, flexibilidad...).

- La existencia del programa presencial en los Centros Madrid Salud, para quien prefiera esta posibilidad. Pinchando en un enlace se accede a la lista completa de Centros Madrid Salud (un total de 14 centros, con turnos de mañana y tarde, distribuidos en otros tantos distritos de la capital), con sus direcciones y teléfonos para inscripciones.

- Los compromisos que el usuario asume una vez inscrito al programa.

Asimismo se incluye un apartado donde se abordan determinadas expectativas inadecuadas que son comunes entre los usuarios. Estas expectativas son modificadas desde el principio porque condicionan de modo importante la evolución del tratamiento. En síntesis, se trata de establecer con claridad que:

- El objetivo del programa es dejar de fumar del todo y para siempre; se invita al usuario a rechazar el objetivo de fumar menos o dejar de fumar sólo durante un tiempo.

- Es necesario que exista una decisión y un compromiso firme con el propósito de dejar de fumar.

- Es necesario un esfuerzo personal; no existen "varitas mágicas" para dejar de fumar.

- Es necesario actuar de modo metódico y planificado; no valen decisiones impulsivas ni tampoco el recurso a las "ganas" o la "fuerza de voluntad".

La sección concluye con una invitación a tomarse unos minutos para decidir, recordándole, además, el compromiso que comporta la decisión de apuntarse. 
En caso de que el usuario decida inscribirse, se pasa al formulario correspondiente.

\section{Bloque 2: inscripción y valoración}

En la pantalla anterior al formulario de inscripción se informa sobre el procedimiento general que se utilizará en la interacción entre el sistema y el usuario: el sistema envía al usuario mensajes de correo electrónico en la fecha prevista en el cronograma; estos mensajes contienen un hipervínculo que, haciendo clic en él, remite a la página web donde, una vez introducidos el nombre de usuario ("nick") y la clave, se accede al contenido correspondiente.

En el formulario de inscripción se recogen únicamente datos demográficos básicos (sexo, edad y nivel de estudios), datos de identificación (un "nick" y una clave de acceso que el usuario debe elegir) y la dirección de correo electrónico.

Se incluye, además, una advertencia de que la cuenta de correo debe estar configurada de tal modo que no exista un filtro anti-spam que pueda impedir la recepción de nuestros mensajes.

Una vez rellenado y enviado el formulario, el sistema comprueba que los datos están completos y que el "nick" y / o la clave no coinciden con las de ningún otro usuario registrado; en este caso se comunica de inmediato al usuario que su inscripción ha sido aceptada y se pasa al formulario de valoración.

El formulario de valoración (figura 1) recoge datos que sirven para decidir la pauta de tratamiento más adecuada, así como las expectativas de autoeficacia del usuario y su situación dentro del modelo de fases del proceso de abandono propuesto por Prochaska y colaboradores (Prochaska y DiClemente, 1992). Específicamente, se recaban los siguientes datos:

- Tipo de tabaco consumido (rubio o negro)

- Concentración de nicotina.

- Test de Fagerstrom

- Existencia de intentos previos de dejar de fumar, procedimientos utilizados y resultados obtenidos.

- Método que prefiere de los dos propuestos para la cesación.

- Consumo de fármacos.

- Expectativas de autoeficacia (probabilidad estimada de conseguir dejar de fumar).

- Fase del proceso de abandono en el modelo de Prochaska y cols.

- Fecha elegida para dejar de fumar o para iniciar la reducción del tabaco (en caso de optar por este método).

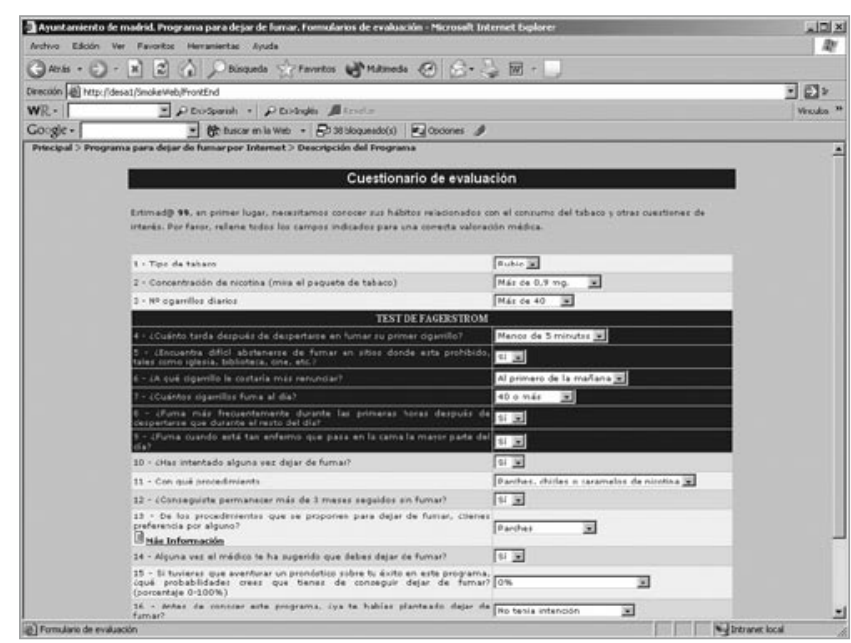

Figura 1. Formulario de valoración.

La pregunta sobre el método preferido contiene un enlace con información sobre cada uno de los métodos (tratamiento psicológico con parches de nicotina, o tratamiento psicológico con reducción gradual).

La fecha elegida para dejar de fumar o iniciar la reducción debe estar contenida en un intervalo entre 10 y 20 días posteriores a la fecha de inscripción. De no ser así, la fecha no es admitida por el sistema. Este intervalo está pensado para evitar decisiones impulsivas ("lo dejo ya, mañana mismo") y por lo tanto no planificadas y con menos probabilidades de éxito, así como demoras excesivamente largas e inconcretas ("dentro de tres meses") donde se pierde el estado motivacional actual.

\section{Plan de intervención}

Una vez remitidos los datos, éstos son procesados por el sistema según un algoritmo de decisión que da como resultado una pauta de tratamiento que se remite de inmediato al usuario con su cronograma completo de contactos con el programa.

Existen cuatro pautas posibles (figura 2):

- Pauta 1: tratamiento psicológico con parches de nicotina a dosis altas

- Pauta 2: tratamiento psicológico con parches de nicotina a dosis estándar.

- Pauta 3: tratamiento psicológico con parches de nicotina a dosis bajas.

- Pauta 4: tratamiento psicológico con reducción gradual de la ingesta de nicotina y alquitrán (RGINA). 


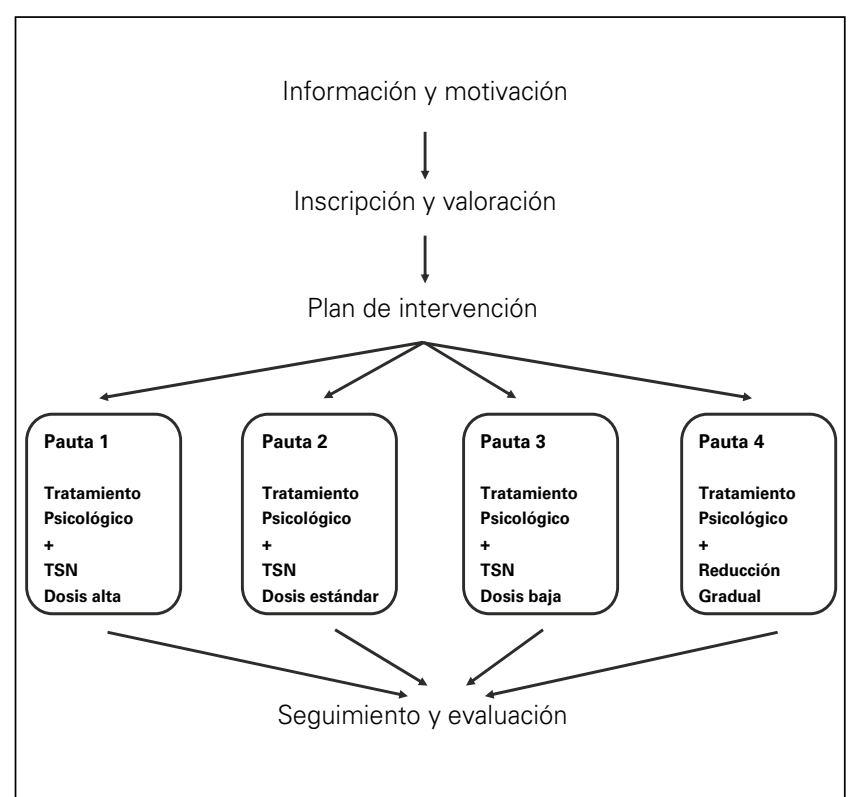

Figura 2. Esquema general del proceso.

Los tipos de parches utilizados y el tiempo de utilización en cada una de las pautas aparecen descritos en la tabla 2. Como se ve, en todas las pautas los parches se utilizan durante 6 semanas.

El algoritmo decide entre una $u$ otra pauta en función de los siguientes parámetros:

- Existencia o no de contraindicaciones para el uso de parches de nicotina.

- Experiencia previa con uno u otro método, y resultados obtenidos. Se asume que si un método ya ha sido utilizado con anterioridad y dio buenos resultados durante algunas semanas (aunque posteriormente se recayera), lo más adecuado es volver a utilizarlo; de no ser así, se consideraría otro método.

- Deseo expresado por el usuario. Se asume que dar al usuario la posibilidad de elegir entre dos métodos mejora su compromiso con el tratamiento y los resultados.

- Nivel de consumo (cantidad de cigarrillos consumidos diariamente, y concentración nicotínica). Se asume que niveles altos constituyen criterio para el uso de terapia sustitutiva de nicotina.

La respuesta al usuario por parte del sistema es inmediata, y contiene varios enlaces desde donde, haciendo clic, se accede a (ver figura 3):

1. Instrucciones sobre el proceso general, sobre el procedimiento específico que se propone para la cesación (parches de nicotina o reducción gradual) y sobre pautas conductuales a seguir durante los primeros días sin fumar (figura 4).

2. Un cronograma con las pautas de uso de los parches o de la reducción gradual mediante cambio de marca. Los días del cronograma se establecen a partir de la fecha elegida por el usuario para dejar de fumar (si usa parches) o empezar la reducción (si usa reducción gradual), y que es el "día 0" del programa.

3. Un cronograma de todo el proceso, con la serie de contactos programados entre el sistema y el usuario.

4. Información resumida, tipo prospecto, sobre los parches.

5. Un hipervínculo para realizar la primera tarea del programa: una lista de beneficios de dejar de fumar.

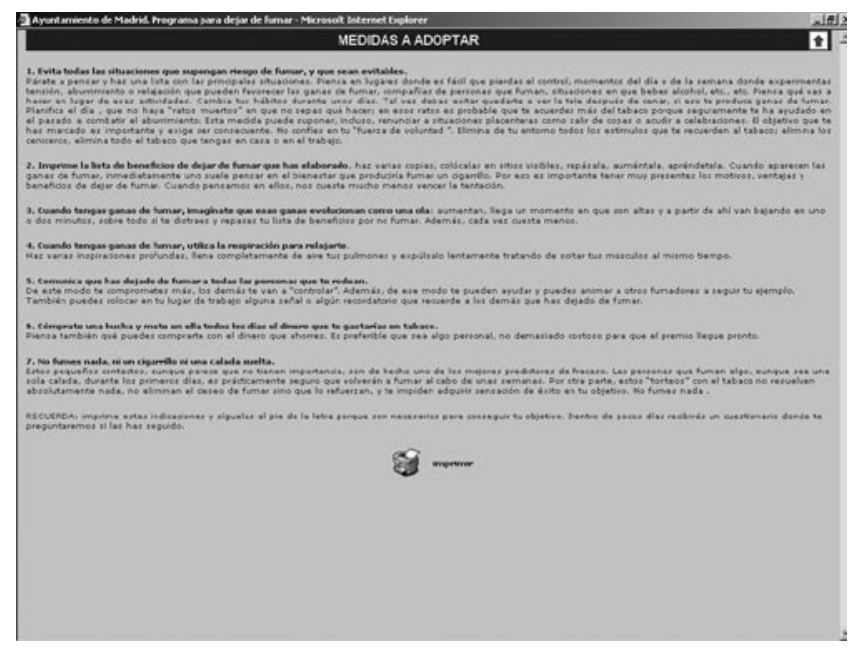

Figura 3. Pantalla que el usuario recibe como respuesta al cuestionario de evaluación, con instrucciones para los primeros días sin fumar, pautas y cronograma completo.

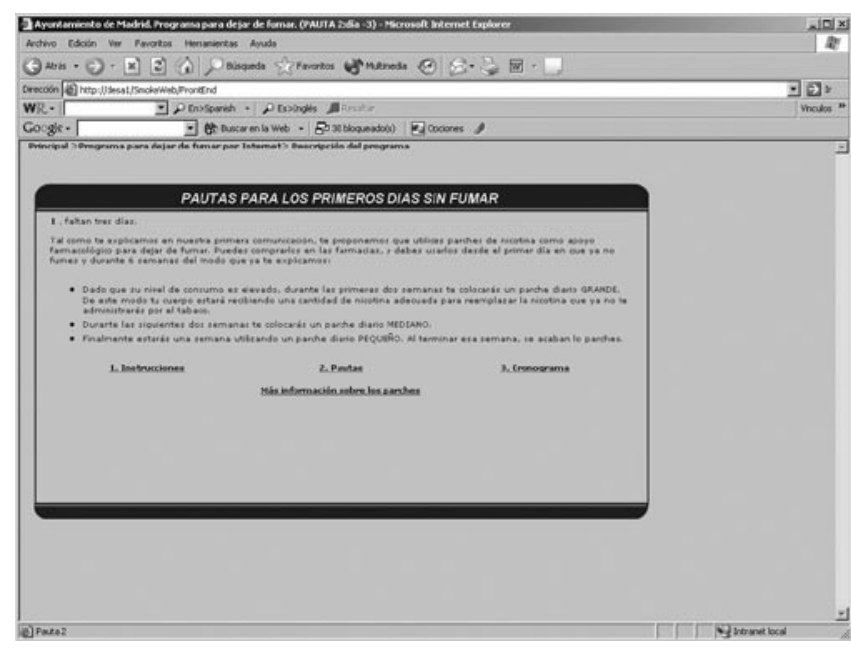

Figura 4. Pautas de conducta para los primeros días. 
Cada una de estas unidades de información se abre en una ventana independiente, y contiene un icono desde el que imprimir el contenido. Se enfatiza la conveniencia de imprimir toda la información, conservarla y, al menos el cronograma, mantenerla en un sitio visible.

Primera tarea: elaboración de una lista de beneficios de dejar de fumar

En la misma sesión en que se realiza la inscripción y valoración, y se reciben todas las instrucciones y el plan, se propone la primera tarea: el usuario debe confeccionar una lista de ventajas o beneficios de dejar de fumar.

El objetivo de esta tarea es incrementar la motivación haciendo consciente al usuario de las indudables ventajas que va a obtener si abandona el tabaco. Pero en lugar de ofrecerle una lista de ventajas para ser leída sin más, exigimos que la lista la elabore él mismo. Sabemos que la participación activa y la toma de decisiones meditada parecen incrementar la efectividad de las estrategias de motivación. Con este fin proponemos una tabla vacía con la opción de imprimir para hacer la lista a mano. Para favorecer que la tarea sea motivadora, añadimos dos ayudas: en primer lugar, la tabla se divide en varias columnas, y en cada una de ellas se deben agrupar ventajas pertenecientes a una categoría ("para mi salud", "para mi calidad de vida", "para la salud de los que me rodean", "para mi economía", "para mi estima personal", "para mi aspecto estético" y "otras ventajas") (figura 5); en segundo lugar, se ofrece una "lista de ejemplos" que pueden ayudar al usuario a ser consciente de más beneficios.

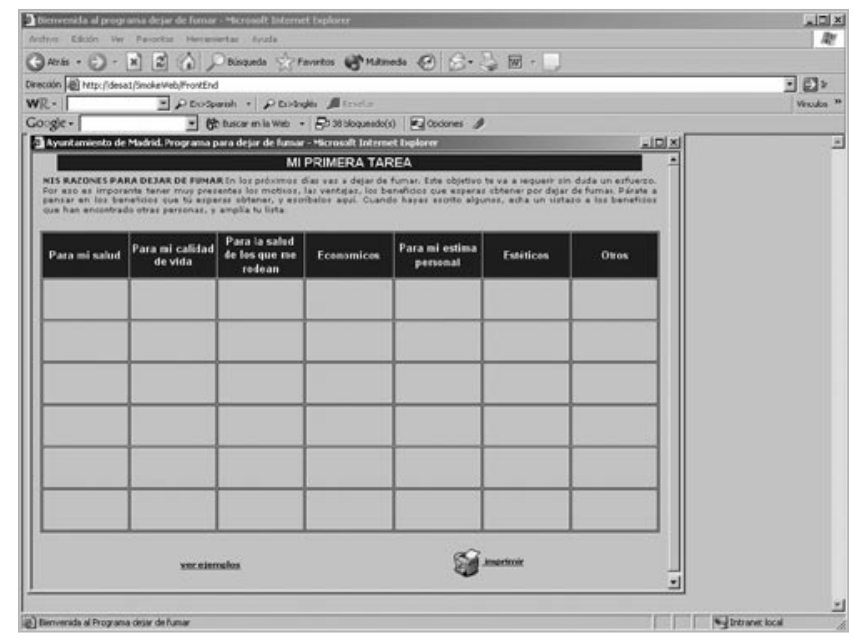

\section{Figura 5. Primera tarea: lista de beneficios de dejar de fumar.}

Posteriormente el usuario debe conservar esta lista, colocarla en un lugar visible, releerla detenidamente y ampliarla en cualquier momento.

\section{Contactos programados entre sistema y usuario}

Con el fin de asegurar que esta información inicial se recibe, y para evitar que se extravíe, es remitida nuevamente en dos ocasiones: cuando faltan 3 días y cuando falta 1 día para la fecha elegida.

Además, es importante tener en cuenta que toda la información que el sistema remite al usuario, tanto la inicial como toda la posterior, es recuperable en cualquier momento. Para ello, el usuario simplemente debe ingresar en la web con su "nick" y su clave, y acceder al cronograma "on line"; una vez allí, haciendo clic en la fecha correspondiente, puede descargar toda la información relativa a esa fecha.

Una vez llegado el "día 0", las interacciones entre sistema y usuario se llevan a cabo según el cronograma. En cada uno de los días señalados, el sistema envía un correo electrónico de aviso al usuario, con un enlace a la web. Una vez allí, el usuario introduce su nick y su clave y accede a la información o cuestionario correspondiente.

Estas interacciones son de varios tipos a lo largo del programa (ver figura 6):

- Instrucciones o pautas que el usuario debe seguir. Aparecen de modo estructurado, breve y sencillo. Se plantean en dos momentos distintos que se corresponden con las dos fases que distinguimos en el abandono. En primer lugar, pautas para los primeros días sin fumar, que el usuario recibe pocos días antes de la fecha elegida y que tienen como objetivo conseguir una abstinencia inicial; en segundo lugar, pautas para mantenerse sin fumar, que se reciben dos semanas después de haber abandonado el tabaco, y que se orientan hacia el mantenimiento de la motivación y la prevención de las recaídas.

- Recordatorios. Consisten en mensajes breves donde se recuerda al usuario que se aproxima la fecha de cambiar el tipo de parche (pautas 1, 2 y 3) o la marca de tabaco (pauta 4) o su abandono.

- Autoevaluaciones. Consisten en listas de comprobación sobre el cumplimiento de cada una de las pautas. El usuario debe limitarse a responder "SI" o "NO" a las preguntas sobre si ha llevado a cabo o no cada una de las indicaciones que ha recibido, y recibe un mensaje corrector (si la respuesta no es la deseable) o reforzante (en caso de que sí lo sea) de modo inmediato. Aunque no se pretende evaluar el cumplimiento, sino sólo recordar al usuario la importancia de hacer las tareas, se adopta un formato de cuestionario o "lista de comprobación" con el fin de requerir la participación activa del usuario y de este modo mejorar el proceso de aprendizaje. Las respuestas a las autoevaluaciones no son registradas (figura 7). 
- Cuestionarios de evaluación. Se cumplimentan al final del programa y a los 3, 6 y 12 meses. Tienen como objetivo conocer los resultados del programa y algunas variables asociadas a ellos (figura 8). Específicamente se pregunta:

- Si fuma o no fuma.

- Si ha ganado peso, y cuánto.
- Sus expectativas de autoeficacia de cara a continuar sin fumar.

- A qué atribuye el continuar sin fumar o el haber recaído.

- Si tiene intención de volver a intentarlo, en caso de haber recaído.

Figura 5. Secuencia de contactos entre sistema y usuario.

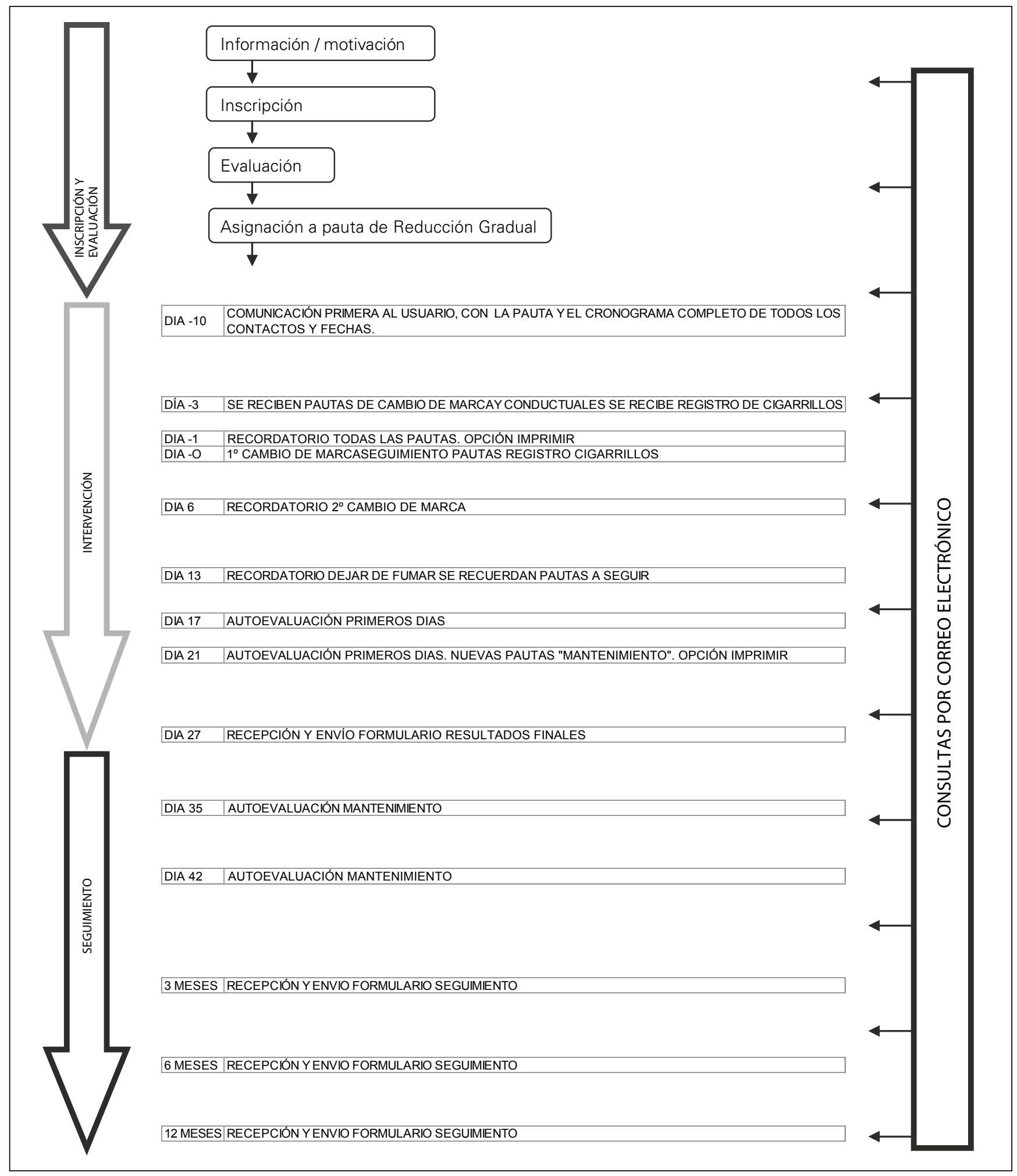




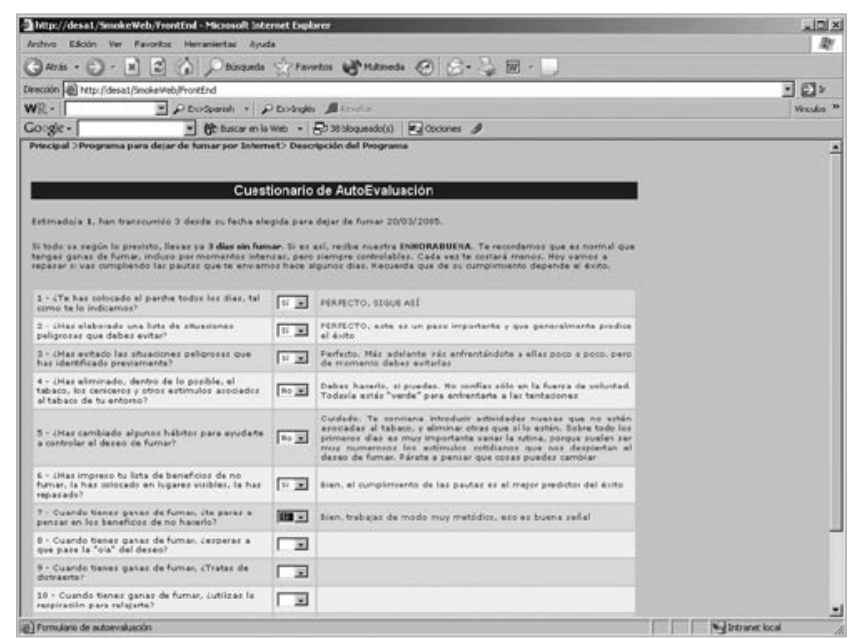

Figura 7. Cuestionario de autoevaluación.

\section{RESULTADOS}

868 sujetos declararon permanecer sin fumar al final del período de tratamiento. Este número representa el $17,8 \%$ de los 4865 sujetos que finalizaron este período sin abandonar y sin ser dados de baja por el sistema por no realizar las tareas durante las primeras 2 semanas (figura 9).

En el seguimiento a los 6 meses únicamente eran evaluables 2816 sujetos, puesto que para el resto aún no habían transcurrido 6 meses desde la fecha de inicio en el momento de redactar este informe. En este momento declararon permanecer sin fumar 289 sujetos, lo que representa un $10,3 \%$ del total (figura 10)

En ambos casos se contabiliza a los individuos que no responden al cuestionario de evaluación como fumadores.

Utilizando el paquete estadístico SPSS-12 se construyó un modelo de regresión logística con el fin de identificar variables predictoras de los resultados. En el análisis se incluyeron todas las variables contenidas en la evaluación inicial. El modelo resultante clasificaba correctamente al 71,4\% de los no fumadores al final del tratamiento, y se componía de cuatro variables:

1. Pauta de tratamiento utilizada, con resultados favorables para quienes utilizaron pautas de tratamiento psicológico con parches de nicotina frente a quienes utilizaron pauta de tratamiento psicológico con reducción gradual $(\mathrm{OR}=0.331)$.

2. Sexo, con resultados favorables para los varones (OR=0.726).

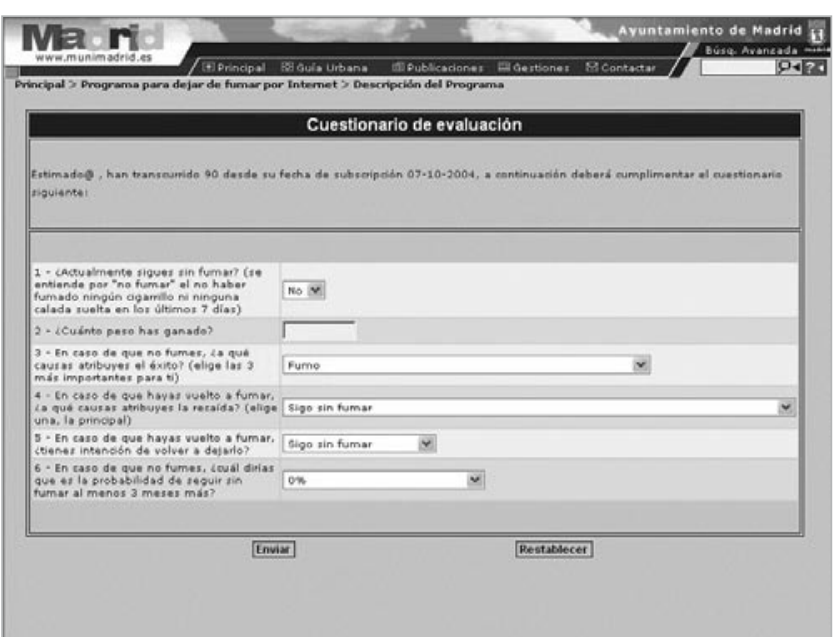

Figura 9. Cuestionario de evaluación.

3. Haber permanecido o no con anterioridad más de 3 meses sin fumar, con resultados favorables para quienes sí habían permanecido (OR=1.271).

4. Expectativas de autoeficacia antes del tratamiento (OR=1,006).

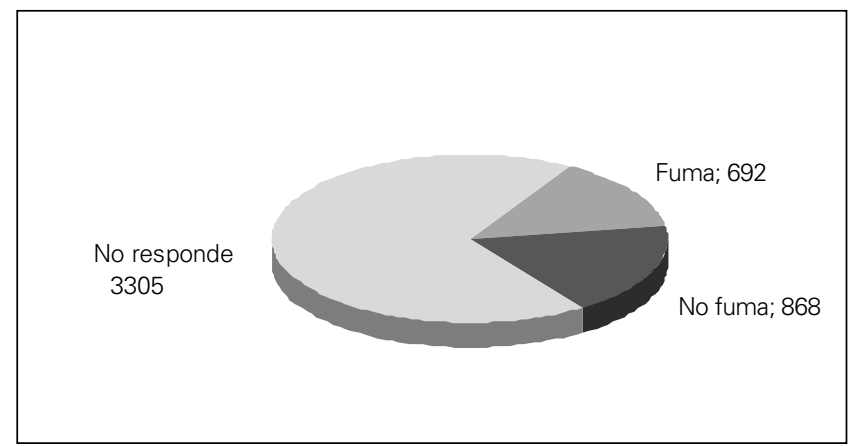

Figura 9. Resultados al final del tratamiento.

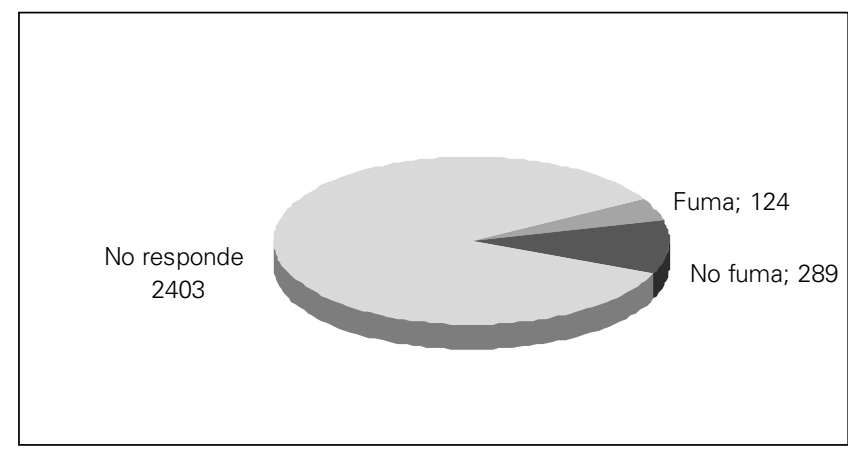

Figura 10. Resultados a los 6 meses de seguimiento. 


\section{DISCUSIÓN}

Según Schwartz (1987) menos del $4 \%$ de los fumadores han participado alguna vez en tratamientos para dejar de fumar cara a cara, que son los que han demostrado mayor eficacia. Por lo tanto, la valoración de los resultados expuestos debe partir de la consideración de que, en un programa dirigido a la erradicación de un comportamiento practicado por más del $30 \%$ de la población, tan importante al menos como conseguir el éxito en las personas que se inscriban es que efectivamente se inscriban muchos sujetos. Este criterio de valoración es similar al que se aplica en el caso de las guías de autoayuda y también, por ejemplo, en el caso del consejo sanitario breve en atención primaria de salud, sin duda una de las estrategias fundamentales contempladas en todos los planes de actuación contra el tabaquismo y donde se prioriza el alcance poblacional frente a la eficacia en sí misma. Por otro lado, esta pretensión de llegar a gran cantidad de beneficiarios potenciales exige eliminar al máximo las posibles barreras en el acceso a los programas; la consecuencia de este enfoque es la participación de una proporción muy elevada de individuos con muy baja motivación, lo cual provoca una elevada proporción de abandonos y fracasos. Se trata de sacrificar la eficacia (entendida como porcentaje de éxitos) en aras de la accesibilidad, algo a menudo no suficientemente tenido en cuenta cuando se determinan los criterios de éxito, excesivamente centrados en el porcentaje de abstinentes.

Por lo tanto, nuestros referentes en el momento de valorar la efectividad de nuestra intervención "on line" no deben ser, como apuntábamos en la introducción, los meros porcentajes de abstinentes obtenidos por programas presenciales. Se trata de líneas de actuación paralelas, complementarias, que se dirigen a objetivos diferentes y cuyos criterios de efectividad son también, por lo tanto, diferentes.

Parece más coherente tomar como referencia el alcance y la efectividad de los procedimientos que utilizan medios de comunicación masivos. Así, las guías de autoayuda impresas ofrecen una eficacia estimada en el $5 \%$ de abstinentes al año (Fiore, Bailey, Cohen y Dorfman, 2000), y en muchos casos dicha estimación es difícil de establecer por no contar con procedimientos de evaluación de los resultados. Puede encontrarse una revisión detallada de la efectividad de los procedimientos de autoayuda, sus subclasificaciones y de sus limitaciones metodológicas en Gil (2003).

Si comparamos nuestros resultados con los ofrecidos por los escasos programas similares que utilizan Internet, éstos son, por ejemplo, notablemente mejores que los encontrados por Schneider, Walter y O'Donnell (1990), que en un programa intensivo e interactivo sobre 1.158 participantes lograron un 7,5 $\%$ o un $17,7 \%$ de abstinentes al mes, dependiendo de si se contaban a los perdidos en el seguimiento como fumadores o si no se les contaba. Téngase en cuenta, por otra parte, que se ha argumentado (Hall et al, 2001) que contabilizar a los usuarios perdidos en el seguimiento como fumadores es un procedimiento excesivamente conservador, puesto que con mucha probabilidad una parte de ellos se mantienen abstinentes.

En un análisis de las diferencias entre nuestro programa y otros aparentemente similares, parece importante considerar su diferente grado de proactividad. Este puede variar desde sistemas en los que cada paso hacia delante depende de la toma de decisiones del usuario sin un plazo temporal específico, es decir, donde se deja completamente que sea el usuario el que tome la iniciativa o no de avanzar en el momento en que él lo decida, hasta programas donde el usuario, una vez inscrito, entra en una secuencia preestablecida de interacciones con el sistema según un calendario previo, que incluye además recordatorios que el sistema envía a través de correo electrónico. Sobre este punto, parecen preferibles procedimientos más directivos o proactivos. Así, en una comparación con los datos presentados en este informe, podrían atribuirse los peores resultados del estudio de Stoddard et al. (2005), donde entre un 3,5 y un 8,3\% del total de inscritos (dependiendo del procedimiento utilizado para la cesación) estaban abstinentes al mes de seguimiento, precisamente a la mayor proactividad de nuestro procedimiento. Obviamente, siempre existe la posibilidad de abandonar el programa si ese es el deseo del usuario. Esta reflexión es consistente con trabajos como el de Lenert et al. (2004), que encuentran mayor efectividad precisamente en procedimientos donde el sistema envía mensajes por correo electrónico, pre-programados en función de la fecha, en lugar de esperar a que sea el usuario el que establezca contacto.

En cuanto a las variables predictoras de los resultados, encontradas en la regresión logística, establecemos las siguientes consideraciones:

- El uso de parches de nicotina de modo conjunto con el tratamiento psicológico ofrece una ods ratio de 0,331 frente a su no utilización. Si tenemos en cuenta que el uso de parches de nicotina implica una inversión económica de cierta importancia para el usuario, en un programa que por otra parte no exige otros compromisos de horarios ni asistencias, cabe plantearse hasta qué punto la efectividad diferencial del procedimiento con parches es atribuible a los parches o a la motivación asociada a su uso. El 
bajo porcentaje de efectividad obtenido por los parches de nicotina por sí solos frente a los tratamientos conductuales cuando se controla la variable del coste del tratamiento para el usuario (véase la revisión de Gil, 2003) hace nos conduce a plantear esta hipótesis. Dirimir esta cuestión implicaría un diseño de asignación aleatoria en dos grupos, sin que existiera coste para el uso de parches.

- Los mejores resultados obtenidos por los varones son coherentes con algunos hallazgos conocidos (Jarvis, 1994; Ward, Klesges, Zbikowski, Bliss y Garvey, 1997; Bjornson, Rand, Connet, Lindgren, Nides, Pope et al., 1995), si bien en otros casos no se han encontrado tales diferencias. En los estudios en que, como en el nuestro, sí se han encontrado diferencias significativas, se ha argumentado que tienen que ver con la diferente relación que tienden a establecer hombres y mujeres con el tabaco, más centrada en el refuerzo positivo en el caso de los varones y en el refuerzo negativo en el caso de las mujeres (Jané, 2004). Aunque no existen conclusiones sobre esta cuestión, las implicaciones terapéuticas de esta hipótesis son evidentes.

- En cuanto a las dos últimas variables predictivas (haber permanecido anteriormente más de 3 meses sin fumar, y las expectativas de autoeficacia), sin duda se trata de dos aspectos íntimamente asociados desde el punto de vista conceptual: las expectativas de autoeficacia se construyen fundamentalmente a partir de la experiencia exitosa previa. En cuanto a su poder predictivo sobre la ejecución exitosa futura, se trata de un postulado básico de la Teoría del Aprendizaje Social (Bandura, 1977).

Finalmente, tan interesante como la consideración de aquellas variables que aparecen como predictivas de los resultados es la consideración de aquellas otras que no aparecen, a pesar de haber sido encontradas como tales en otros trabajos o haber sido postulado su valor predictivo desde determinados modelos teóricos. Particularmente, no encontramos valor predictivo en la intensidad del consumo (puntuación en test de Fagerstrom) ni en la fase del proceso de cambio en que se encuentra el fumador dentro del modelo de Prochaska y cols. (Prochaska y DiClemente, 1992).

La consideración de la fase del proceso de cambio en que se encuentra el fumador ha sido señalada en los últimos tiempos como elemento clave porque condicionaría los objetivos y las estrategias a seguir en la clínica. Así, se dice que, con fumadores en fase de precontemplación o contemplación, el objetivo terapéutico debe consistir en todos los casos en favorecer el avance en el proceso de motivación hasta que, en una fase posterior, llegue el momento de plantearse la abstinencia. Aunque sin duda este planteamiento tiene el valor, entre otros, de subrayar el valor intrínseco de la intervención previa al momento de tomar la decisión de abandonar el tabaco, y de la intervención motivacional como estrategia fundamental, implica un sesgo cognitivista discutible teóricamente (¿la motivación y la decisión anteceden necesariamente a la actuación? ¿Acaso la motivación no nace de la actuación misma?). Así, Costa y López (2006) plantean precisamente la idoneidad de una estrategia orientada a hacer que el sujeto tenga fácil el decidir pasar a la acción lo antes posible, desde la hipótesis de que el verdadero motor motivacional es la misma actuación y su relación con los resultados. En esta misma línea, recientemente West y Sohal (2006), en una encuesta con 918 fumadores que habían intentado dejar de fumar al menos una vez, y 996 exfumadores, encuentran mejores resultados precisamente en aquellos intentos de abandono que no habían sido precedidos de un proceso de planificación, esto es, cuyos actores no se encontraban en la "fase adecuada" según el modelo de Prochaska y cols. Estos hallazgos y estas consideraciones encierran implicaciones terapéuticas de cierto calado habida cuenta de la popularidad que el modelo de Prochaska y cols. y sus ha alcanzado como referente teórico en contextos como la atención primaria de salud en nuestro país. En todo caso, no es este el lugar para extendernos sobre la cuestión.

\section{CONCLUSIONES}

Aunque se trata de un estudio exploratorio y no de una investigación controlada (así, en particular, el uso del parche de nicotina hace difícil establecer la atribución exacta de los resultados del tratamiento psicológico), es posible avanzar algunas conclusiones:

1. El uso de internet en la promoción de comportamientos saludables como el abandono del tabaco es probablemente más coste-efectivo que las guías tradicionales en soporte de papel, y cuenta frente a estas con indudables ventajas.

2. En el caso de programas que implican una serie de contactos prolongada en el tiempo es más efectivo el uso de procedimientos proactivos, es decir, aquellos en que una vez inscrito el usuario es el sistema el que toma la iniciativa de contactar con él según una pauta preestablecida en cuanto a fechas y contenidos, frente a aquellos en los que, al menos en algún momento, la posibilidad de continuar depende de la iniciativa del usuario.

3. Es más eficaz el uso de procedimientos que plantean al usuario tareas específicas cuyo cumplimien- 
to es recordado y supervisado, y no meramente recomendaciones generales.

4. La experiencia previa en intentos de abandono del tabaco modifica la probabilidad de tener éxito en el intento presente, por su efecto sobre las expectativas de autoeficacia.

5. El uso de terapia sustitutiva de nicotina de modo combinado con indicaciones de comportamiento mejora la eficacia de éstas; no está claro qué papel cumple la motivación asociada al hecho de asumir el coste económico del fármaco.

6. No encontramos evidencia que apoye el que los sujetos clasificables como "precontempladores" o "contempladores" deban demorar en todos los casos la fecha de dejar de fumar.

7. Algunas posibles mejoras de programas como el expuesto incluyen el uso de procedimientos más flexibles y adaptables a las características del usuario o a colectivos especiales, el uso paralelo de foros o chats que promuevan el apoyo mutuo entre usuarios o la inclusión de material multimedia.

\section{REFERENCIAS}

Bandura, A. (1977). Self-efficacy: Toward a unifying theory of behavioral change. Psychological Review, 84, 191-215.

Becoña, E. (1994). Tratamiento del tabaquismo. En: J. L. Graña (Ed.), Conductas adictivas. Teoría, evaluación y tratamiento. Madrid: Debate.

Becoña, E. (1997). Programa para dejar de fumar. Junta de Andalucía.

Becoña, E. y Vázquez, F. L. (2001). Effectiveness of personalized written feedback through a mail intervention for smoking cessation: A randomisedcontrolled trial in Spanish smokers. Journal of Consulting and Clinical Psychology, 69, 33-40.

Bjornson, W., Rand, C., Connet, J. E., Lindgren, P., Nides, M., Pope, F., et al. (1995). Gender differences in smoking cessation after 3 years in the Lung Health Study. American Journal of Public Health, 85, 223-230.

Comité Nacional para la Prevención del Tabaquismo (2001). Guía de procedimientos para ayudar a los fumadores a dejar de fumar. Adicciones, 13, 211-216.

Copeland, J., y Martin, G. (2004). Web-based interventions for substance use disorders: a qualitative review. Journal of Substance Abuse Treatment, 26, 109-116.

Costa, M. y López, E. (2006). Dar poder para vivir. Fortalecer a las personas en la intervención psicológica. Pirámide.

Fenichel, M., Suler, J., Barak, A., Zelvin, E., Jones, G., Munro, K., Meunier, V., Walker-Schmucker, W. (2002). Myths and realities of online clinical work. CyberPsychology \& Behavior, 5, 481-497.

Fiore, M., Novotny, T., Pierce, J., Giovino, G., Hatziandreu, E., Newcomb, P., Surawicz, T., y Davis, R. (1990).
Methods used to quit smoking in the United States. Do cessation programs help? Journal of American Medical Association, 263, 2760-2765.

Fiore, M. C., Bailey, W. C., Cohen, S. J., Dorfman, S. F., Goldstein, M. G., Gritz, E. R., et al. (2000). Treating tobacco use and dependence. Rockville, MD: U. S. Department of Health and Human Services, Public Health Service.

Gil Roales-Nieto, J. (2003). Guía de tratamientos psicológicos eficaces en el tabaquismo. En: Pérez, M., Fernández, J. R., Fernández, C., Amigo, I., (Eds.), Guía de tratamientos psicológicos eficaces // (pp. 287-353). Madrid: Pirámide.

Hajek, P. y Stead, L. F. (2003). Aversive smoking for smoking cessation. En The Cochrane Library, Issue 4. Chichester, RU: Wiley and Sons, Ltd.

Hall, S. M., Delucchi, K. L., Velicer, W. F., Kahler, C. W., Ranger-Moore, J., Hedeker, D., Tsoh, J. y Niarua, R. (2001). Statistical analysis of randomised trials in tobacco treatment: Longitudinal designs with dichotomous outcome. Nicotine Tobacco Research, 3, 193-202.

Jané, M. (2004). Mujeres y tabaco: aspectos principales específicos de género. Adicciones, 16 (Supl. 2), 115130.

Jarvis, M. J. (1994). Gender differences in smoking cessation: Real or myth? Tobacco Control, 3, 324-328.

Lancaster, T. y Stead, L.F. (2003a). Individual behavioural counselling for smoking cessation (Cochrane Review). En The Cochrane Library, Issue 4. Chichester, RU: Wiley and Sons, Ltd.

Lancaster, T. y Stead, L.F. (2003b). Self-help interventions for smoking cessation (Cochrane Review). En The Cochrane Library, Issue 4. Chichester, RU: Wiley and Sons, Ltd.

Lancaster, T., Stead, L. F. (2005). Asesoramiento conductual individual para el abandono del hábito de fumar (Revisión Cochrane traducida). En: La Biblioteca Cochrane Plus, 3. Oxford: Update Software Ltd.

Lenert, L., Muñoz, R. F., Perez, J. E. y Bansod, A. (2004). Automated e-mail messaging as a tool for improving quit rates in an Internet smoking cessation intervention. Journal of the American Medical Informatics Association, 11, 235-240.

Míguez, M. C., Vázquez, F. L. y Becoña, E. (2002). Effectiveness of telephone contact as an adjunct to a self-help program for smoking cessation. A randomised controlled trial in Spanish smokers. Addictive Behaviors, 27, 139-144.

Miguez, M. C. (2004). Los procedimientos de autoayuda para dejar de fumar. Adicciones, 16 (Supl. 2), 339-358.

Ministerio de Sanidad y Consumo (2003). Encuesta Nacional de Salud 2003. Recuperado el 15 de Marzo de 2006 de www.msc.es/estadEstudios/estadisticas/ encuestaNacional/home.htm

Ministerio de Sanidad y Consumo y Comité Nacional para la Prevención del Tabaquismo (2005). Se puede dejar de fumar. Claves para conseguirlo. Recuperado el 15 de Marzo de 2006 de http://www.msc.es/ciudadanos/ proteccionSalud/adolescencia/docs/guiaTabaco.pdf. 
Prochaska, J. O. y DiClemente, C. C. (1992). Stages of change in the modification of problem behaviors. En: Hersen, M., Eisler, R. M., y Miller, P. M. (Eds.) Progress in Behavior Modification (pp.184-214). Sycamore, IL: Sycamore Publishing Company.

Raw, M., McNeill, A. y West, R. (1998). Smoking cessation guidelines for Health Professionals. A guide to effective smoking cessation interventions for the health care system. Thorax, 53 (Supl. 5), 1-19.

Schneider, S. J., Walter, R. y O’Donnell, R. (1990). Computerized communication as a medium for behavioral smoking cessation treatment: controlled evaluation. Computers in Human Behavior, 6, 141-151.

Schwartz, J.L. (1987). Review and evaluation of smoking cessation methods: The United States and Canada, 1978-1985. Washington, DC: U.S. Department of Health and Human Services.

Silagy, C., Lancaster, T., Stead, L., Mant, D., Fowler, G. (2005). Terapia de reemplazo de nicotina para el abandono del hábito de fumar (Revisión Cochrane traducida). En: La Biblioteca Cochrane Plus, 3. Oxford: Update Software Ltd.

Stead, L. F., Lancaster, T. (2005). Programas de terapia conductual grupal para el abandono del hábito de fumar (Revisión Cochrane traducida). En: La Biblioteca Cochrane Plus, 3. Oxford: Update Software Ltd.

Stoddard, J. L., Delucchi, K. L., Muñoz, R. F., Collins, N. M., Pérez Stable, E. J., Augustson, E., Lenert, L. L. (2005). Smoking cessation research via the Internet: a feasibility study. Journal of Health Communication, 10, 27-41.

Tsoh, J. Y. y McClure, J. B. (1997). Smoking cessation 2: components of effective intervention. Behavioral Medicine, 23, 15-27.

Ward, K. D., Klesges, R. C., Zbikowski, S. M., Bliss, R. E., Garvey, A. J. (1997). Gender differences in the outcome of an unaided smoking cessation attempt. Addictive behaviours 22, 521-33. 